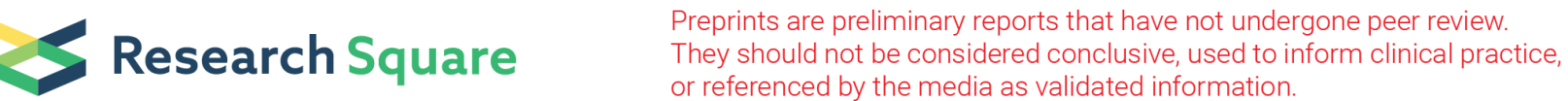

\section{Novel method using DW-MRI and ADC images to guide stereotactic biopsy for the diagnosis small primary angiitis of the central nervous system: $A$ case report}

\section{Xu Su}

Jilin University No 3 Hospital: China-Japan Union Hospital of Jilin University

\section{Liang Han}

Jilin University No 3 Hospital: China-Japan Union Hospital of Jilin University

Mengxing Li

Beijing Tiantan Hospital

\section{Zhengming Wang}

Jilin University No 3 Hospital: China-Japan Union Hospital of Jilin University

Jiadui Gao

Jilin University No 3 Hospital: China-Japan Union Hospital of Jilin University

\section{Yu Tian}

Jilin University No 3 Hospital: China-Japan Union Hospital of Jilin University

Chao Du ( $\square$ duchao@jlu.edu.cn )

Jilin University No 3 Hospital: China-Japan Union Hospital of Jilin University https://orcid.org/00000001-8804-1971

\section{Case report}

Keywords: Case report, Biopsy guiding method, DW-MRI/ADC map, Primary angiitis of the central nervous system, Diagnosis

Posted Date: May 3rd, 2021

DOI: https://doi.org/10.21203/rs.3.rs-202210/v2

License: (1) This work is licensed under a Creative Commons Attribution 4.0 International License. Read Full License 


\section{Abstract}

Objective To determine the role of diffusion-weighted magnetic resonance imaging (DW-MRI) and apparent diffusion coefficient (ADC) imaging to guide stereotactic biopsy for the diagnosis of intracranial angiitis.

Case presentation In a 28-year-old woman who had experienced inactive headache and right limbs numbness for 4 days, preoperative magnetic resonance (MR) scanning, enhanced scanning, diffusion tensor imaging, magnetic resonance spectroscopy, diffusion-weighted imaging (DWI), and ADC image scanning were performed. Stereotactic biopsy was performed in one target where the area of edema detected with MR FLAIR, and two targets where the area shown as a high-value and a lower-value area in the DWI/ ADC image. Pathological examinations together with computed tomographic and enhanced MRI scans were conducted after surgery. A preoperative enhanced MRI scan showed a uniform lowintensity lesion in the patient's left centrum semiovale, with a volume of $3.1 \mathrm{~cm}^{3}$. The DWI and ADC images showed uneven high-intensity signals and different ADC values in the lesion area, respectively. During surgery, tissues around the lesion and the lesion center were sampled at the three selected targets. The postoperative pathological diagnosis was primary angiitis of the central nervous system, and the patient was given anti-inflammatory medication and hormone therapy. The 3-year follow-up confirmed that the patient had recovered well, with a Glasgow Outcome Scale score of 5.

Conclusion DW-MRI and ADC images can be reliably used to determine the location of small intracranial lesions, and guide stereotactic biopsy to facilitate the diagnosis of primary vasculitis of the central nervous system.

\section{Introduction}

Primary angiitis of the central nervous system (PACNS) is an idiopathic inflammatory syndrome confined to the brain parenchyma, spinal cord, and leptomeninges, that mainly involves the vascular walls ${ }^{1}$. The incidence rate of PACNS is estimated to be $2-3$ cases per 1 million individuals ${ }^{2,3}$. A Mayo Clinic study of 163 patients with PACNS, published in 2015, is still the most extensive sample of the disease yet reported 4,5 . The etiology of PACNS is not yet clear. The peak age of onset is around 50 years, and it may be slightly more prevalent in women than in men. It often affects young people who lack common risk factors for cerebrovascular disease 1,2,6. The clinical manifestations of PACNS are diverse, and the specificity of laboratory tests and imaging is poor, so its diagnosis is challenging. Because the treatment of PACNS differs from the treatments required for other CNS diseases, it is vital to make an accurate diagnosis as early as possible.

The diagnosis of PACNS is challenging, and a definite diagnosis can only be made using biopsy findings after brain surgery ${ }^{7-14}$ or stereotactic biopsy ${ }^{15}$. However, there are no reports of specialized methods based on radiological features to guide stereotactic biopsy for the diagnosis of PACNS. Here, we report a 
case with a small PACNS diagnosed with stereotactic biopsy of targets identified by diffusion-weighted magnetic resonance imaging (DW-MRI) and apparent diffusion coefficient (ADC) mapping.

\section{Case Report}

A 28-year-old woman who had experienced inactive headache and right limbs numbness with progressive aggravation for 4 days was admitted to our hospital on June 26, 2017. She had experienced no fever since symptom onset. She demonstrated clear consciousness with accurate verbal responses. Physical examinations indicated her right upper limb muscle strength was grade III and her right lower limb muscle strength was grade IV in Manual Muscle Testing Scale. Her right hand could maintain grip, but her fingers had trouble completing delicate motor tasks. There was no clinical history of any relevant CNS, neuronal, or muscles related illness.

Serological tests performed on admission revealed a white blood cell (WBC) of $7.38 \times 10^{9} / \mathrm{L}$ (normal range, $4.0-10.0 \times 10^{9} / \mathrm{L}$ ), percentage of neutrophils of $59.5 \%$ (normal range, $50-70 \%$ ), percentage of lymphocytes of $31.3 \%$ (normal range, 15-70\%), percentage of monocytes of $7.6 \%$ (normal range, 3.0$10.0 \%$ ), neutrophil count of $4.39 \times 10^{9} / \mathrm{L}$ (normal range, 2.0-7.5 × 109/L), lymphocyte count of $2.31 \times$ $10^{9} / \mathrm{L}$ (normal range, $0.8-4.0 \times 10^{9} / \mathrm{L}$ ), and monocyte count of $0.56 \times 10^{9} / \mathrm{L}$ (normal range, $0.3-0.8 \times$ $\left.10^{9} / \mathrm{L}\right)$.

Two days after admission, MRI showed an area of inhomogeneous hypointense signal on a T1-weighted image (T1WI) and a hyperintense signal on T2-weighted image (T2WI) and T1-weighted fluid-attenuated inversion recovery (FLAIR) images, with a size of $15 \times 17 \times 22 \mathrm{~mm}^{3}$ (volume, $3.1 \mathrm{~cm}^{3}$ ), surrounded by edema, within the left centrum semiovale (Fig. 1). A contrast-enhanced MRI (CE-MRI) scan showed a lesion within the left centrum semiovale, with apparent inhomogeneous enhancement and an irregular shape, whereas the surrounding edema showed no enhancement (Fig. 2). Magnetic resonance angiography (MRA) showed no apparent anomalies (Fig. 3). DW-MRI and ADC mapping showed an area of inhomogeneous hyperintensity within the left centrum semiovale. The core of the hyperintense signal on DWI was hypointense on the ADC map (Fig. 4). Magnetic resonance diffusion tensor imaging (DT-MRI) showed that parts of the white matter fiber tracts were interrupted and identified an area of lower fractional anisotropy (FA) within the left centrum semiovale (Fig. 5). Magnetic resonance spectroscopy (MRS) images showed that the content of choline-containing compounds (Cho) and its ratio to creatine (Cr) were increased in this area. In contrast, the content of $N$-acetyl aspartate (NAA) was slightly reduced within the volume in this area: $\mathrm{NAA} / \mathrm{Cr}=2.54, \mathrm{Cho} / \mathrm{Cr}=1.62, \mathrm{Cho} / \mathrm{NAA}=1.22$ (Fig. 6). The imaging results suggested a strong likelihood of brain glioma.

At day 3 of admission, an analysis of her cerebrospinal fluid (CSF; three tubes were collected, $2 \mathrm{ml}$ per tube) showed colorless CSF with a pressure of $100 \mathrm{mmH}_{2} \mathrm{O}$ (normal range, $80-180 \mathrm{mmH}_{2} \mathrm{O}$ ). The patient's WBC count was $1 \times 10^{6} / \mathrm{L}$ (normal range, $0-8 \times 10^{6} / \mathrm{L}$ ) and her protein, glucose, and chlorine levels in the CSF were $0.31 \mathrm{~g} / \mathrm{L}$ (normal range, $0.12-0.60 \mathrm{~g} / \mathrm{L}$ ), $3.40 \mathrm{mmol} / \mathrm{L}$ (normal range, 2.2-3.9 
$\mathrm{mmol} / \mathrm{L}$ ), and $121.4 \mathrm{mmol} / \mathrm{L}$ (normal range, $120.0-132.0 \mathrm{mmol} / \mathrm{L}$ ), respectively. The samples were negative for Pandy's reaction. CSF cytology revealed no exfoliated cells.

The radiological diagnosis were initially consistent with angiitis, low-grade glioma, or metastatic carcinoma. On day 10, the patient underwent stereotactic biopsy with Komai's stereotactic instrument (Mizuho Medical Innovation, Tokyo, Japan) (Fig. 7). The area of edema around the lesion in the left centrum semiovale was initially targeted, and $2 \times 2 \times 2 \mathrm{~mm}^{3}$ of white soft tissue was sampled. The second and third biopsies targeted areas on the DWI/ADC map with different characteristics, and sampled two $2 \times 2 \times 3 \mathrm{~mm}^{3}$ regions of gray, slightly toughened tissue. A CT scan of the head performed 1 day after stereotactic surgery showed the presence of gas (evident as a shadow) in the left centrum semiovale (Fig. 7D).

Pathological analysis of the biopsies returned a diagnosis of primary angiitis of the central nervous system (Fig. 8). Therefore, she was prescribed an anti-inflammatory medication (cefazolin powder, $2 \mathrm{~g}$ twice daily by intravenous injection) and hormone therapy (hydroprednisone, $50 \mathrm{mg}$ twice daily by intravenous injection).

Ten days after surgery, the patient's right upper limb muscle strength was grade IV and her right lower limb muscle strength was grade $V$ in Manual Muscle Testing Scale. At 15 days after surgery, her right upper limb muscle strength was grade $\mathrm{V}$. The patient's symptoms of limbs have improved significantly. A follow-up MRI examination of the head was conducted 2 months after stereotactic surgery. MRI showed a small residual cavity of hypointensity on T1WI and hyperintensity on T2WI and FLAIR with a clear boundary in the left centrum semiovale after surgical treatment, with no enhancement (Fig. 9). A followup MRI examination of the head 18 months after stereotactic surgery showed that the small cavity in the left centrum semiovale had disappeared. The 3-year follow-up confirmed that the patient had recovered well, with a Glasgow Outcome Scale score of 5.

\section{Discussion}

The methods used to diagnose PACNS primarily include CSF examination, imaging, and pathological investigation. Pathology plays a vital role in the diagnosis of PACNS, whereas imaging plays an auxiliary role to the pathological examination. If no pathological examination is made, PACNS can only be an exclusionary diagnosis $1,6,15$.

Imaging is extremely valuable in the diagnosis of PACNS. As well as the traditional technologies of CT, MRI, and cerebrovascular imaging, such as computed tomography angiography (CTA), MRA, and digital subtraction angiography (DSA), the diagnosis of PACNS with various other imaging techniques has been

reported, including new molecular imaging procedures ${ }^{16-18}$. Most PACNS patients have various MRI abnormalities ${ }^{19}$, but some PACNS patients have normal angiograms ${ }^{20}$. MRA and DSA examinations of PACNS patients can detect stenosis or dilation of multisegment blood vessels in ischemic lesions, but rarely find long, complete vascular occlusions or aneurysm-like changes ${ }^{1,6}$. Until recently, DSA was the 
most widely used technique to diagnose PACNS ${ }^{6,18,21}$. The most typical manifestations of PACNS on MRI are multiple asymmetric ischemic lesions involving the bilateral cerebral hemispheric cortex, subcortical structure, and deep white matter, accompanied by intracerebral or subarachnoid hemorrhage. Other rare manifestations include hemorrhage-like, mass-like, or cyst-like lesions in the brain parenchyma, which are challenging to distinguish from other cerebrovascular diseases, demyelination diseases, and brain tumors ${ }^{6,22}$. Patients with a solitary mass-like PACNS are relatively rare $(<5 \%)$, and the differential diagnosis is more difficult ${ }^{1}$. The literature also reports that DWI and DTI examinations can detect PACNS patients with negative MRI results ${ }^{6,23}$, and DWI sequences can detect acute ischemic lesions ${ }^{21}$. In study a 16 patients, 15 showed DWI hyperintensity and nine of these also showed hypointensity in the middle of the lesion. All 16 patients underwent a brain biopsy (a stereotactic procedure in 10 and open-wedge surgery in six), which showed lymphocytic angiitis in the majority of patients (15/16) and necrotizing angiitis in one patient ${ }^{24}$. In one patient with PACNS with a tumor-like mass lesion, there was no evidence of acute infarction on $\mathrm{DWI}{ }^{25}$.

Tissue biopsy is the key to diagnosing PACNS ${ }^{26}$, and the role of pathological biopsy in the diagnosis of PACNS is irreplaceable. Brain biopsy is now considered the 'gold standard' strategy for the definitive diagnosis of PACNS ${ }^{19}$ and should be pursued not only because it provides information that establishes the diagnosis but also effectively excludes other similar diseases 7,20,21,27. Imaging methods cannot detect some cases of PACNS involving peripheral blood vessels. However, even when imaging reveals typical PACNS manifestations, the biopsy results may not support PACNS diagnose ${ }^{6}$. Pathology results confirm that the inflammation associated with PACNS usually manifests as transmural damage to the involved vessel wall and immune cell infiltration. Thickening of the vessel wall can cause vascular stenosis, disturbing the microcirculation. Simultaneous rupture of the vessel wall and secondary intracranial hemorrhage can occur due to the fragility of the vessel wall ${ }^{6}$.

Areas of CT or MR enhancement, or areas of abnormal MRS are often been used to guide stereoscopic biopsy and localize intracranial lesions. A framed stereotactic device biopsy, positioning by CT-enhanced, MR-enhanced T1, T2-weighted imaging and MRA-enhanced imaging, was used to diagnose a case of PACNS ${ }^{15}$. The diagnosis rate can reach $80 \%$ when imaging is used to assist pathological biopsy ${ }^{6,22}$. In one study, the classic angiographic features of angiitis were associated with biopsy or postmortem confirmation in only $4.6 \%$ of patients ${ }^{28}$ (32/701), and positive angiography was not correlated with positive biopsy results in a study of 34 PACNS patients ${ }^{29}$. The diagnosis rate of PACNS achieved with surgical biopsy ranged from $36-83 \%$ in previous studies ${ }^{13,15}$.

The surgical biopsy complication rate is $16 \%$, which includes serious complications such as cerebral hemorrhage and epilepsy ${ }^{13,15}$, whereas stereotactic biopsy is less traumatic. It can be used in critically ill patients, and its complication rate is $<13 \%$, with patients experiencing only transient or minor complications ${ }^{15}$. Therefore, the diagnosis rate of pathological biopsy should be increased as possible for the accurate diagnosis and appropriate treatment of patients with PACNS. Because the number of cases 
is small and clinical experience of this disease is limited, the process of PACNS biopsy has not been standardized. The leptomeninges, cortex, and deep white matter must be sampled simultaneously to improve the diagnostic biopsy rate ${ }^{13,15}$.

The low incidence of PACNS and its high heterogeneity means that there is a lack of randomized controlled trials of its treatment ${ }^{6}$. Glucocorticoids alone or in combination with cyclophosphamide can achieve satisfactory results ${ }^{22}$. However, it was reported that the administration of azathioprine, methotrexate, or mycophenolate mofetil as maintenance therapies can achieve more satisfactory results 27.

Our patient had a small intracranial lesion (with a volume of $3.1 \mathrm{~cm}^{3}$ ). MRI and MRA examinations showed that the left vertebral artery was narrow, consistent with the imaging features reported in the literature ${ }^{23}$. Limaye et al. reported that when two patients $(20 \%)$ underwent MRS, the Cho/NAA ratios in both patients were approximately $0.9^{19}$. The result of MRS in this patient $(C h o / N A A=1.22)$ does not have a noticeable prompting effect on the judgment of the lesion's nature. We considered that, because the MR T2 and FLAIR features of PACNS patients can be either normal or abnormal, they could have abnormal DWI and ADC images. Therefore, we performed comprehensive imaging examinations before performing stereotactic biopsy in our patient. The imaging examination allowed areas with different characteristics on the DWI and ADC maps to be selected as multiple targets for stereotactic biopsy, which allowed us to make a precise pathological diagnosis. Follow-up for 3 years confirmed that the patient recovered well after treatment (Glasgow Outcome Scale of 5).

\section{Conclusion}

The diagnosis and treatment of this patient suggest that for patients suspected of PACNS, presetting the puncture targets for stereotactic biopsy according to various features on DW-MRI and ADC maps will improve the diagnosis rate of diagnostic biopsy surgery. Imagings assist target localization, and can used to support stereotactic biopsy for the diagnosis of small intracranial lesions in patients with PACNS.

\section{Abbreviations}

MR: magnetic resonance; MR-DWI: diffusion-weighted magnetic resonance imaging; ADC: apparent diffusion coefficient image; DTI: diffusion tensor imaging; 1H-MRS: magnetic resonance spectroscopy; CT: computyed tomography; GOS: Glasgow Outcome Scale; PACNS: Primary angiitis of the central nervous system; WBC: white blood cell; MR CE: Contrast enhanced magnetic resonance; MRA: Magnetic resonance angiography; FA: fractional anisotropy; Cho: choline-containing compounds; Cr: creatine; NAA: $\mathrm{N}$-acetyl aspartate; CSF: cerebrospinal fluid; CTA: Computed tomography angiography; DSA: Digital subtraction angiography; SAH: subarachnoid hemorrhage.

\section{Declarations}


Acknowledgments

The authors thank the patient for agreeing to participate in her report and for providing her detailed medical history.

\section{Authors' contributions}

Xu Su, Liang Han, Mengxing Li, Zhengming Wang, Jiadui Gao: Data Collection, Manuscript writing. Yu Tian, Chao Du: Manuscript editing. The author(s) read and approved the final manuscript.

\section{Funding}

No funding.

\section{Availability of data and materials}

Not applicable.

\section{Ethics approval and consent to participate}

Ethical approval and patient consent were acquired. All ethical approval and consent procedures were approved by the Institutional Review Board of China-Japan Union Hospital of Jilin University.

\section{Consent for publication}

Not applicable.

\section{Competing interests}

The authors declare no conflict of interest/competing interests in publishing the present manuscript.

\section{References}

1. Lucke M, Hajj-Ali RA. Advances in Primary Angiitis of the Central Nervous System. Current Cardiology Reports. 2014;16(10).

2. Giannini C, Salvarani C, Hunder G, Brown RD. Primary central nervous system vasculitis: pathology and mechanisms. Acta Neuropathologica. 2012;123(6):759-72.

3. Salvarani C, Brown RD, Calamia KT, et al. Primary central nervous system vasculitis: analysis of 101 patients. Annals of Neurology. 2007;62(5):442-51.

4. Berlit P, Kraemer M. Cerebral vasculitis in adults: what are the steps in order to establish the diagnosis? Red flags and pitfalls. Clinical Experimental Immunology. 2014;175(3):419-24.

5. Salvarani C, Brown RD, Christianson T, et al. An Update of the Mayo Clinic Cohort of Patients With Adult Primary Central Nervous System Vasculitis Description of 163 Patients. Medicine. 2015;94:21. 
6. Beuker C, Schmidt A, Strunk D, et al. Primary angiitis of the central nervous system: diagnosis and treatment. Therapeutic Advances in Neurological Disorders. 2018;11.

7. Caputi L, Erbetta A, Marucci G, et al. Biopsy-proven primary angiitis of the central nervous system mimicking leukodystrophy: A case report and review of the literature. Journal of Clinical Neuroscience. 2019;64:42-4.

8. Elbers J, Halliday W, Hawkins C, Hutchinson C, Benseler SM. Brain biopsy in children with primary small-vessel central nervous system vasculitis. Annals of Neurology. 2010;68(5):602-10.

9. Magaki S, Gardner T, Khanlou N, Yong WH, Salamon N, Vinters HV. Brain biopsy in atypical dementia and primary angiitis of the central nervous system-reply. Human Pathology. 2016;51:147-8.

10. Miller DV, Salvarani C, Hunder GG, et al. Biopsy Findings in Primary Angiitis of the Central Nervous System. The American Journal of Surgical Pathology. 2009;33(1):35-43.

11. Saettele MR, Loskutov A, Sigley MJ, Lowe LH, Nielsen DB. Biopsy-proven case of childhood primary angiitis of the central nervous system presenting with bilateral panuveitis and anisocoria. Pediatric Radiology. 2014;45(2):291-5.

12. Safouris A, Stricker J, Michotte A, Voumvourakis K, Gazagnes M-D, Tsivgoulis G. Biopsy-proven fulminant primary angiitis of the central nervous system with normal arteriography: a challenging diagnosis of recurrent ischemic strokes. Neurological Sciences. 2013;35(1):135-7.

13. Torres J, Loomis C, Cucchiara B, Smith M, Messé S. Diagnostic Yield and Safety of Brain Biopsy for Suspected Primary Central Nervous System Angiitis. Stroke. 2016;47(8):2127-9.

14. Yaari R, Anselm IA, Szer IS, Malicki DM, Nespeca MP, Gleeson JG. Childhood primary angiitis of the central nervous system: Two biopsy-proven cases. The Journal of Pediatrics. 2004;145(5):693-7.

15. Stoecklein VM, Kellert L, Patzig M, et al. Extended stereotactic brain biopsy in suspected primary central nervous system angiitis: good diagnostic accuracy and high safety. Journal of Neurology. 2020.

16. Backhaus P, Roll W, Beuker C, et al. Initial experience with [18F]DPA-714 TSPO-PET to image inflammation in primary angiitis of the central nervous system. European Journal of Nuclear Medicine Molecular Imaging. 2020;47(9):2131-41.

17. Boulouis G, de Boysson H, Zuber M, et al. Primary Angiitis of the Central Nervous System. Stroke. 2017;48(5):1248-55.

18. Thaler $C$, Kaufmann-Bühler A-K, Gansukh T, et al. Neuroradiologic Characteristics of Primary Angiitis of the Central Nervous System According to the Affected Vessel Size. Clinical Neuroradiology. 2017;29(1):37-44.

19. Suthiphosuwan S, Bharatha A, Hsu CCT, et al. Tumefactive Primary Central Nervous System Vasculitis: Imaging Findings of a Rare and Underrecognized Neuroinflammatory Disease. American Journal of Neuroradiology. 2020;41(11):2075-81.

20. Bhattacharyya S, Berkowitz AL. Primary angiitis of the central nervous system: avoiding misdiagnosis and missed diagnosis of a rare disease. Practical Neurology. 2016;16(3):195-200. 
21. Limaye K, Samaniego EA, Adams HP. Diagnosis and Treatment of Primary Central Nervous System Angiitis. Current Treatment Options in Neurology. 2018;20(9).

22. Lee Y, Kim J-h, Kim E, et al. Tumor-mimicking primary angiitis of the central nervous system: initial and follow-up MR features. Neuroradiology. 2009;51(10):651-9.

23. Aviv RI, Benseler SM, Silverman ED, et al. MR imaging and angiography of primary CNS vasculitis of childhood. American Journal of Neuroradiology. 2006;27(1):192-9.

24. Wang Q, Wang Z, Wang K, Zheng K, Qi X. Characteristics of mass lesion presentation of primary angiitis of the central nervous system: A single center 11-year retrospective case-series study. Clinical Neurology and Neurosurgery. 2020;199.

25. Huang NFWJ, Lu LY, Liao WC, Su CS. Cerebellar PACNS in an Elderly Patient Present as a Tumor-Like Mass Lesion: A case Report. Acta Neurol Taiwan. 2019;28(2):38-43.

26. Spence S, Ng D, Casault C. Atypical presentation of fulminant primary central nervous system angiitis. Journal of Neuroimmunology. 2019;330:1-4.

27. de Boysson $\mathrm{H}$, Parienti $\mathrm{J}-\mathrm{J}$, Arquizan $\mathrm{C}$, et al. Maintenance therapy is associated with better long-term outcomes in adult patients with primary angiitis of the central nervous system. Rheumatology. 2017;56(10):1684-93.

28. Ferghal McVerry MGM, Peter MRCP, McCarron PhD, Keith W, Muir MD, Mark O. McCarron, MD. Diagnostic test results in primary CNS vasculitis A systematic review of published cases. Neurol Clin Pract. 2017;7(3):10.

29. Raghavan A, Wright JM, Huang Wright C, et al. Concordance of angiography and cerebral biopsy results for suspected primary central nervous system vasculitis: A multi-center retrospective review. Clinical Neurology and Neurosurgery. 2019;185.

\section{Figures}



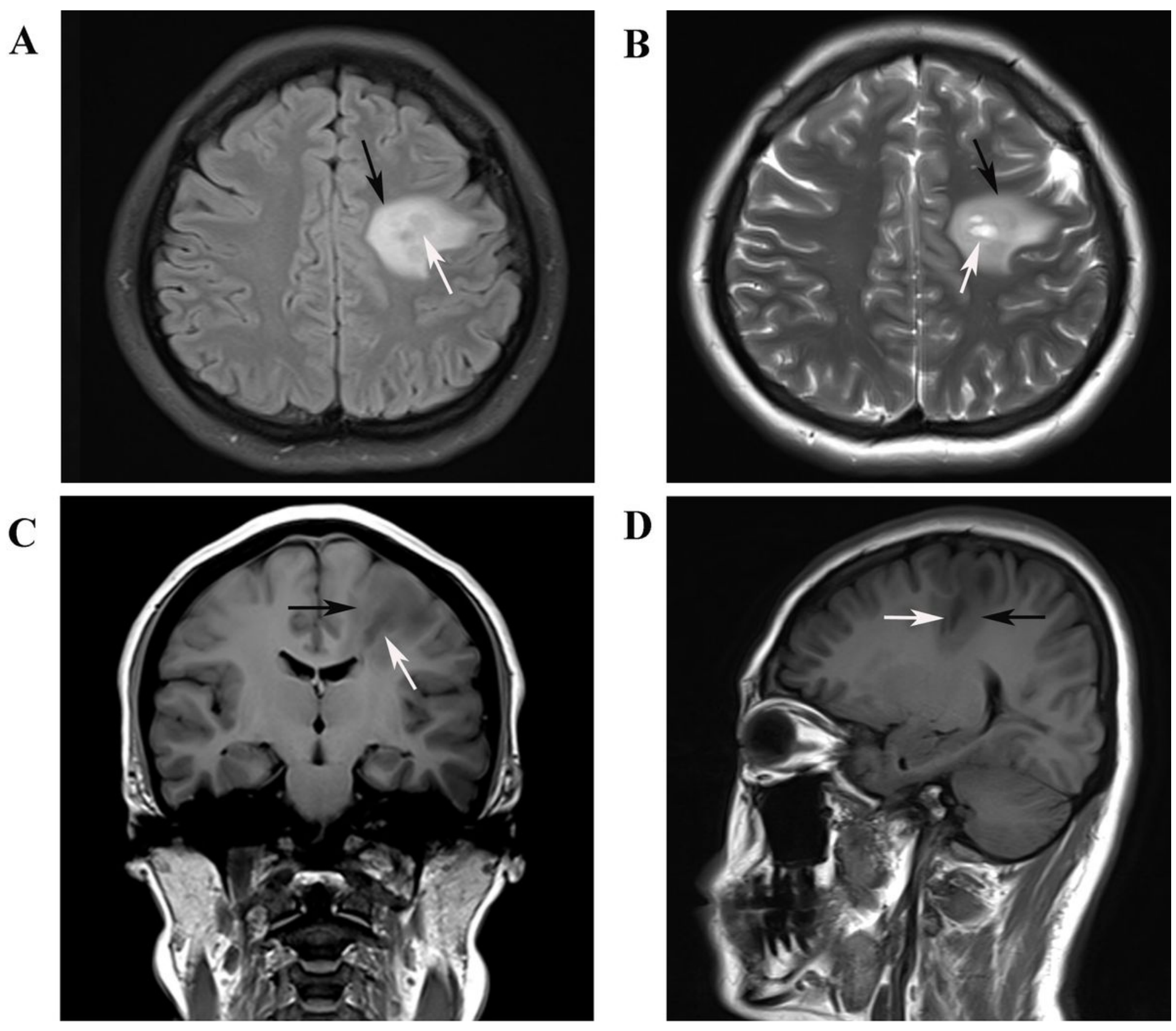

Figure 1

MR images before surgery show an area of inhomogeneous hypointensity on a T1-weighted image (T1WI) and hyperintensity on a T2-weighted image (T2WI) and T1-weighted fluid-attenuated inversion recovery (FLAIR) image, with a size of $15 \times 17 \times 22 \mathrm{~mm} 3$ (volume, $3.1 \mathrm{~cm} 3$ ), surrounded by edema, within the left centrum semiovale (white arrows point to the center of focus; black arrows point to the region of edema). (A) T1-FLAIR axial view. (B) T2WI axial view. (C) T1WI coronal view. (D) T1WI sagittal view. 

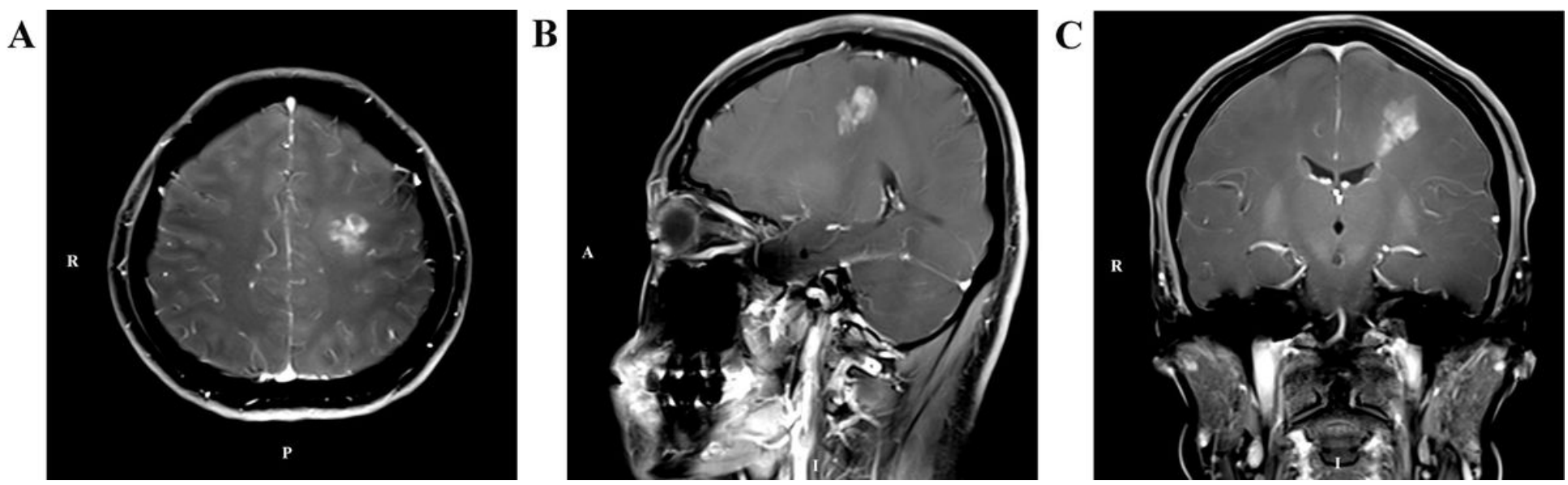

Figure 2

Before surgery, contrast-enhanced T1-weighted magnetic resonance imaging showed a lesion within the left centrum semiovale, with apparently inhomogeneous enhancement and an irregular shape. Edema showed no enhancement. (A) Axial view. (B) Sagittal view. (C) Coronal view.
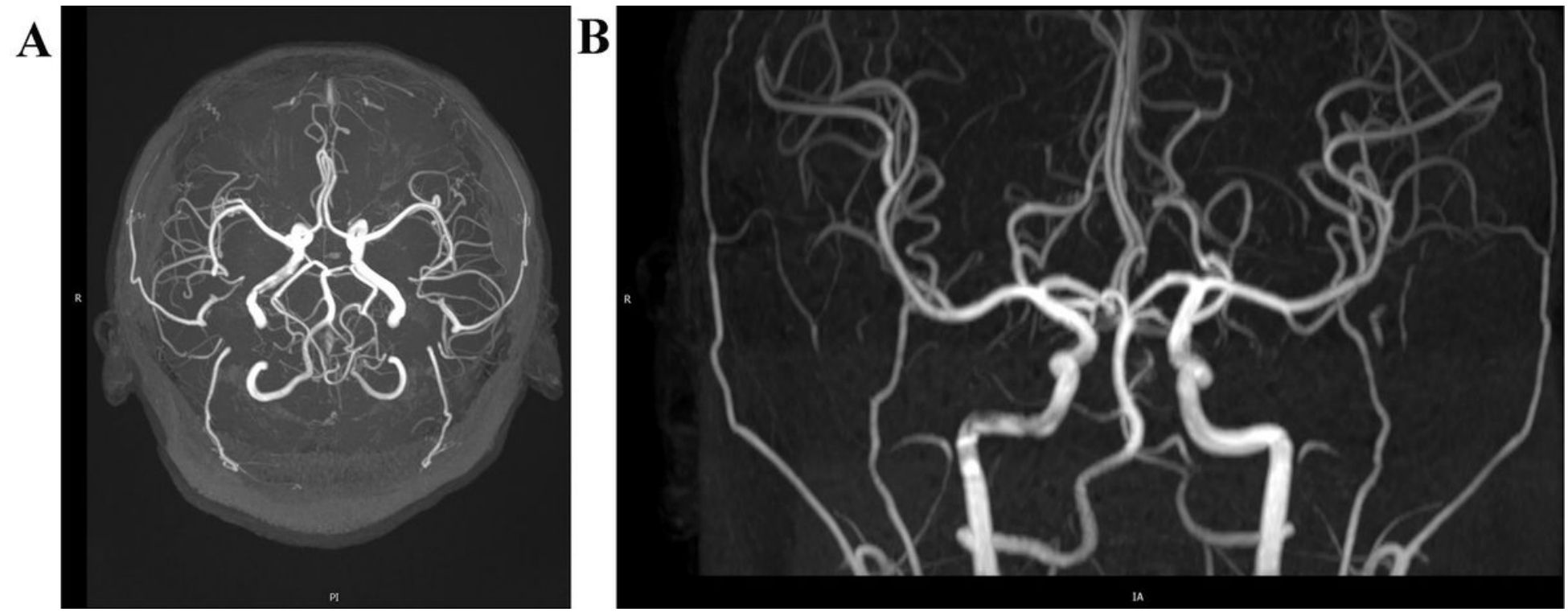

\section{Figure 3}

Before surgery, magnetic resonance angiography (MRA) images showed no obvious anomalies. (A) MRA maximum-intensity-projection (MIP) superior image. (B) MRA-MIP anterior image. 

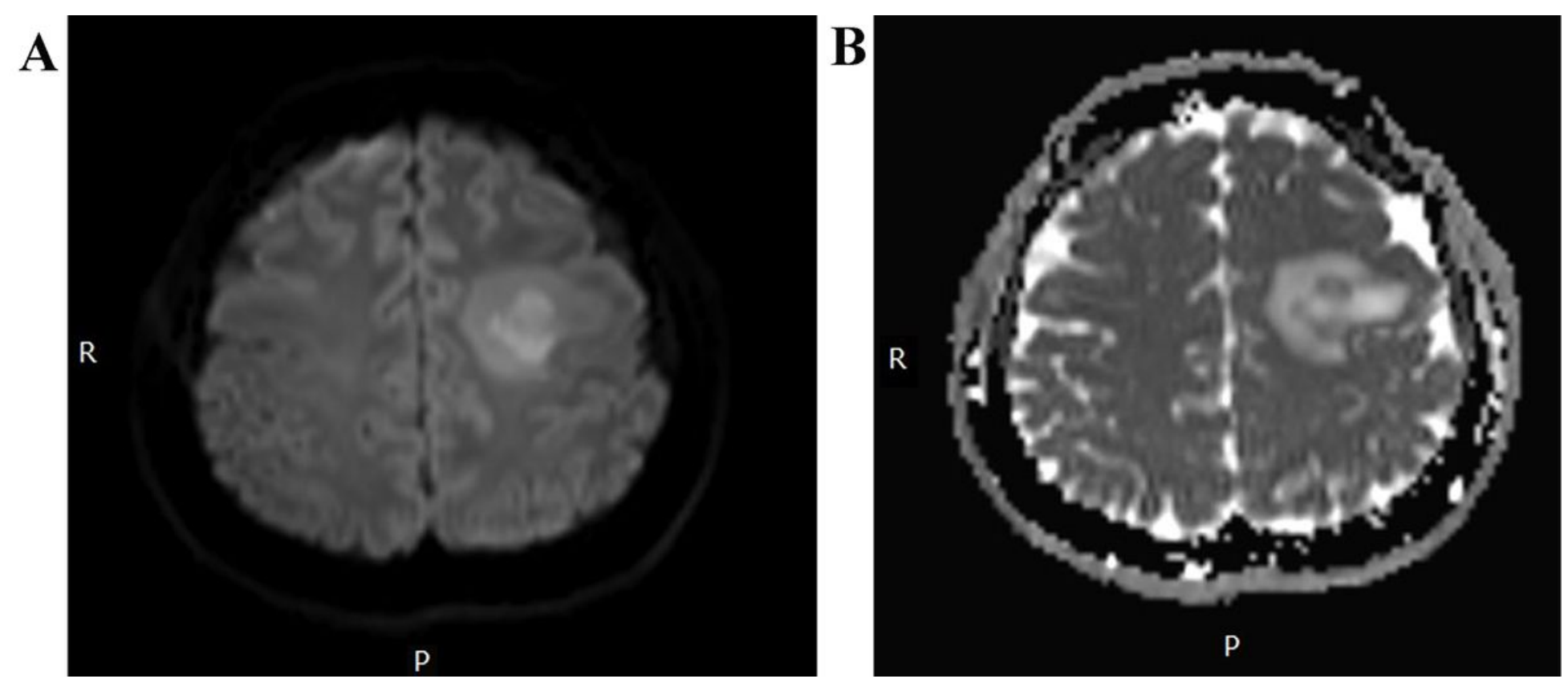

Figure 4

Before surgery, diffusion-weighted magnetic resonance imaging (DW-MRI) and apparent diffusion coefficient (ADC) mapping showed an inhomogeneous hyperintense area within the left centrum semiovale center. DW-MRI revealed a core of hyperintensity, which was hypointense on the ADC map. (A) DWI image $(b=1000 \mathrm{~s} / \mathrm{mm} 2)$. (B) ADC map. 

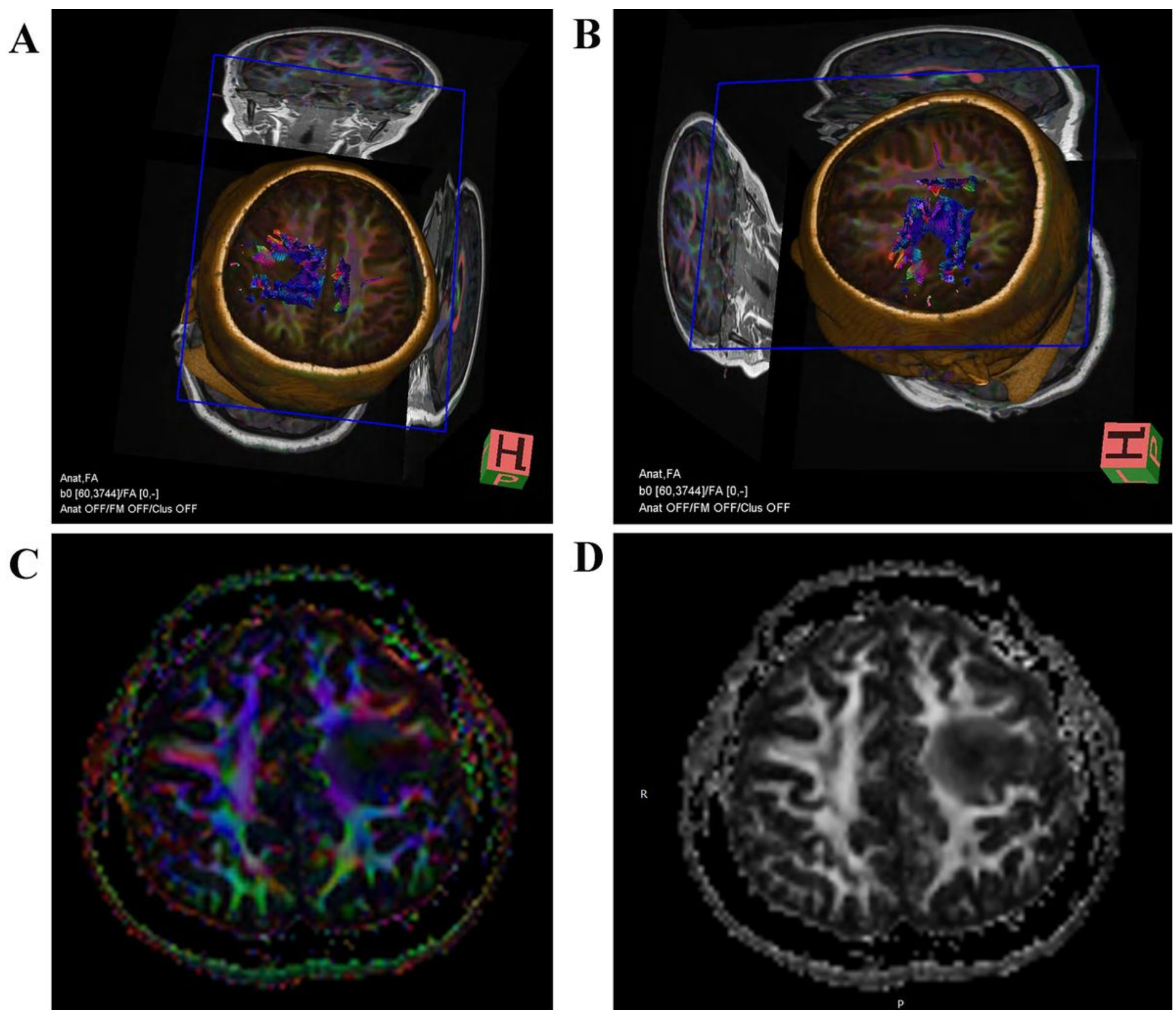

\section{Figure 5}

. Before surgery, magnetic resonance diffusion tensor images showed that parts of the white matter fiber tracts were interrupted and an area of lower fractional anisotropy (FA) within the left centrum. ( $A$ and $B$ ) Diffusion tensor tractography (DTT). (C) FA pseudocolor image. (D) FA gray-scale image. 

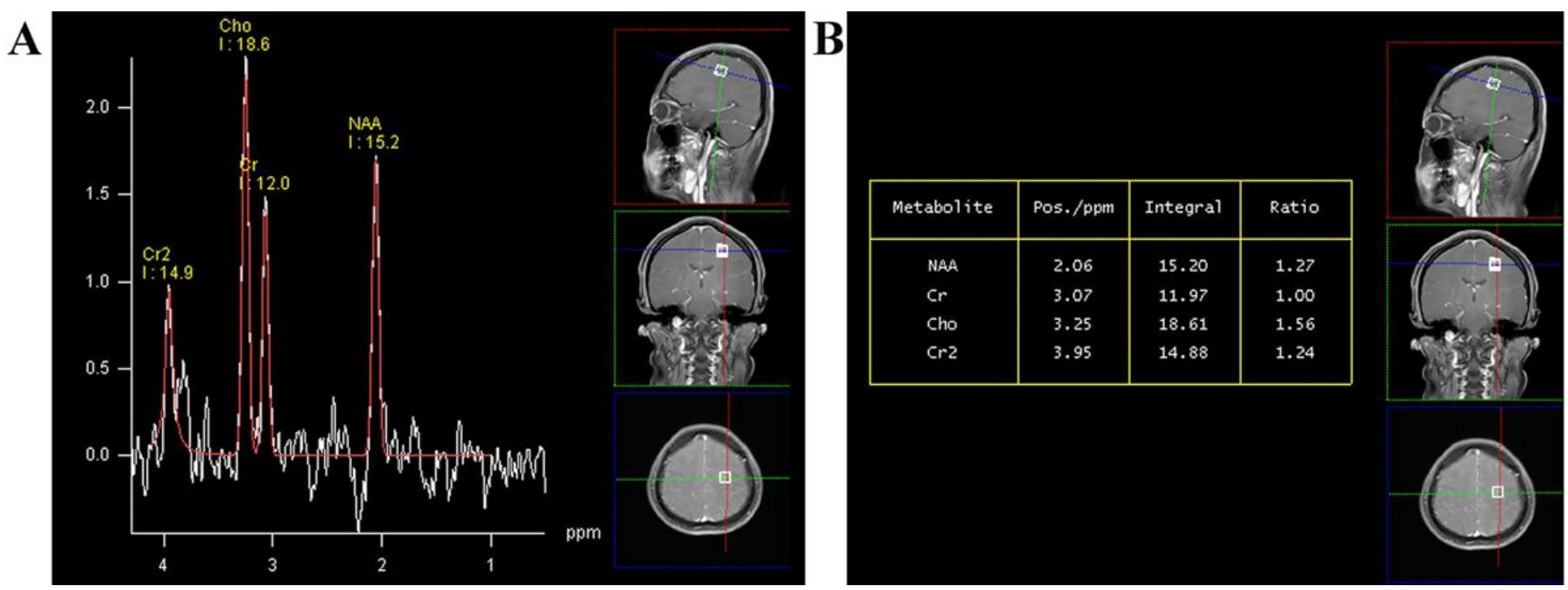

\section{Figure 6}

In single-volume spectroscopy-magnetic resonance spectroscopy (SVS-MRS) images before surgery, the MRS content of choline (Cho) and the Cho/creatine ( $\mathrm{Cr}$ ) ratio were increased, whereas the N-acetyl aspartate (NAA) content declined slightly within the same area. (A) MRS graph of each metabolite and the locations of each single volume. (B) Table of each metabolite and their ratios to $\mathrm{Cr}$. 

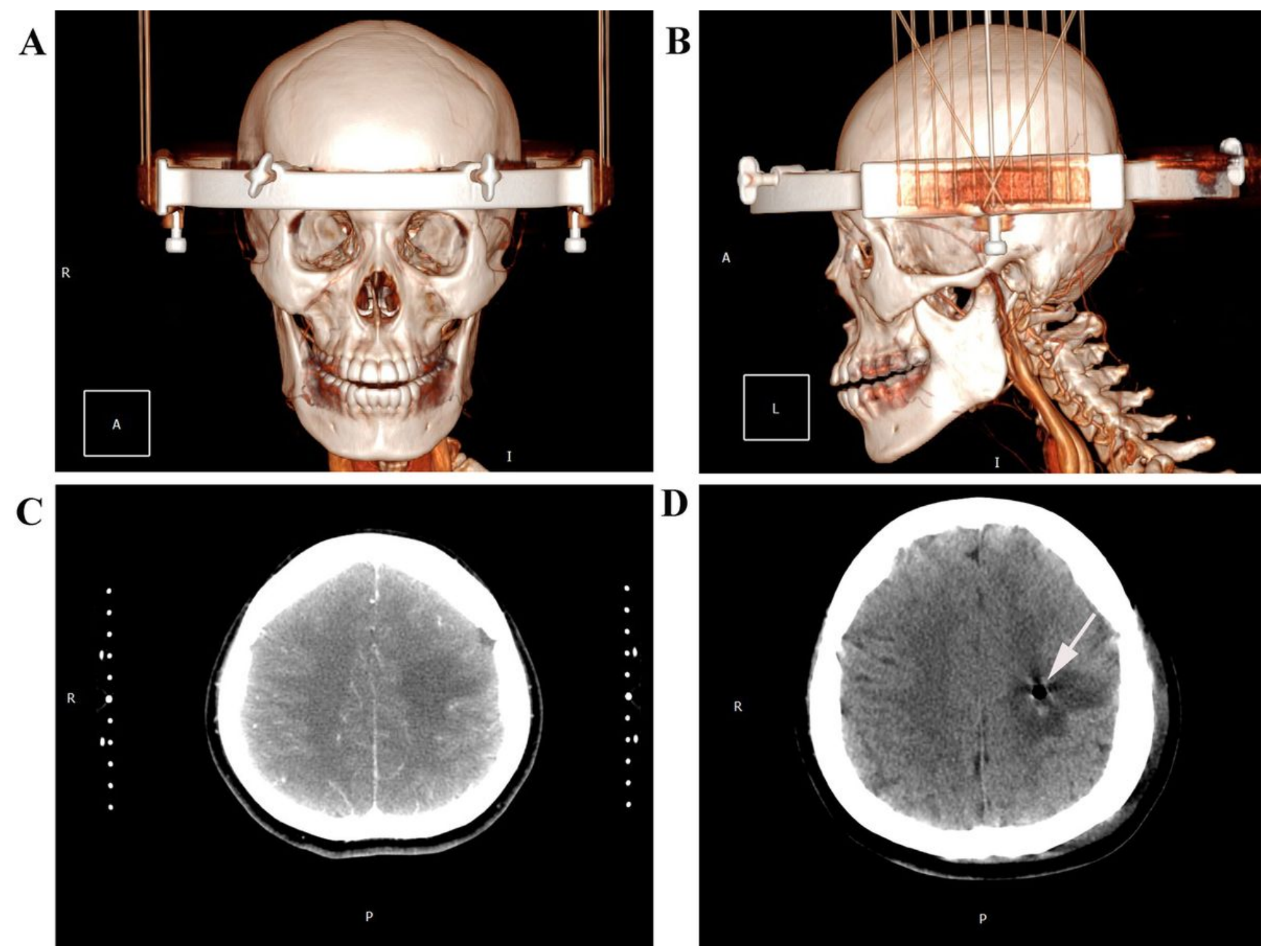

Figure 7

Three-dimensional (3D) computed tomography (CT) images before and after stereotactic biopsy surgery. (A) Localization of the area for stereotactic surgery and an axial image of the 3D CT reconstruction. (B) Stereotactic biopsy localization and sagittal image of the 3D-CT reconstruction. (C) Stereotactic biopsy targeting the left frontal lesion. (D) CT scan of the head, 1 day after stereotactic biopsy, showed a shadow indicating gas present in the left frontal lesion (white arrow). 

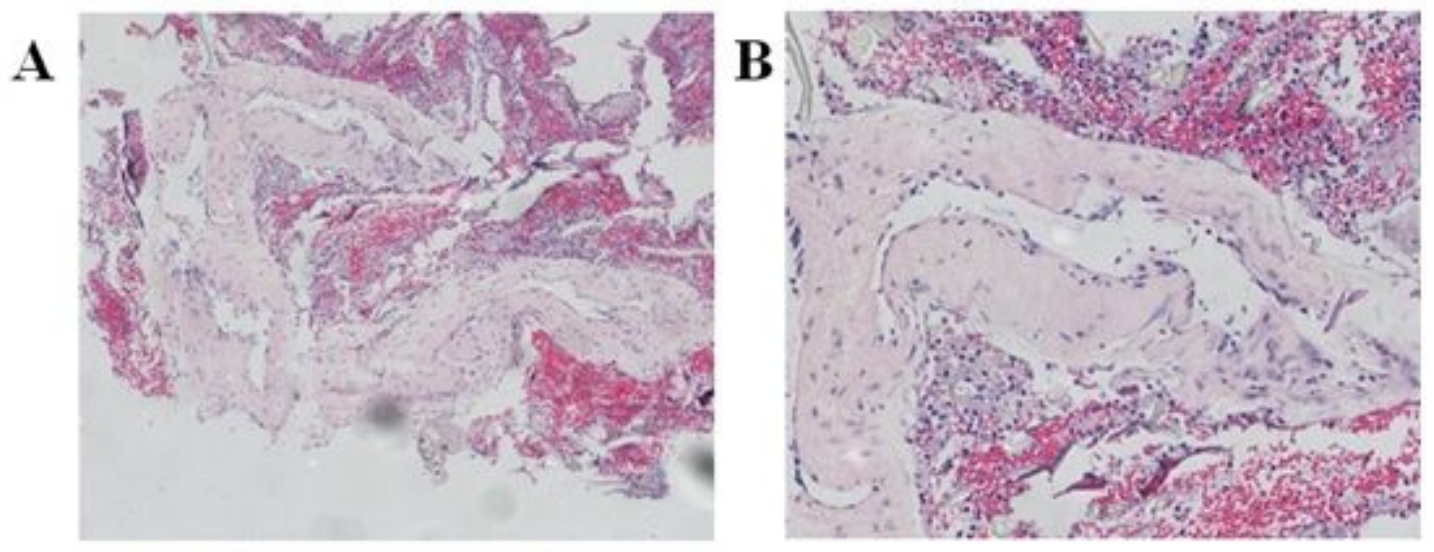

C
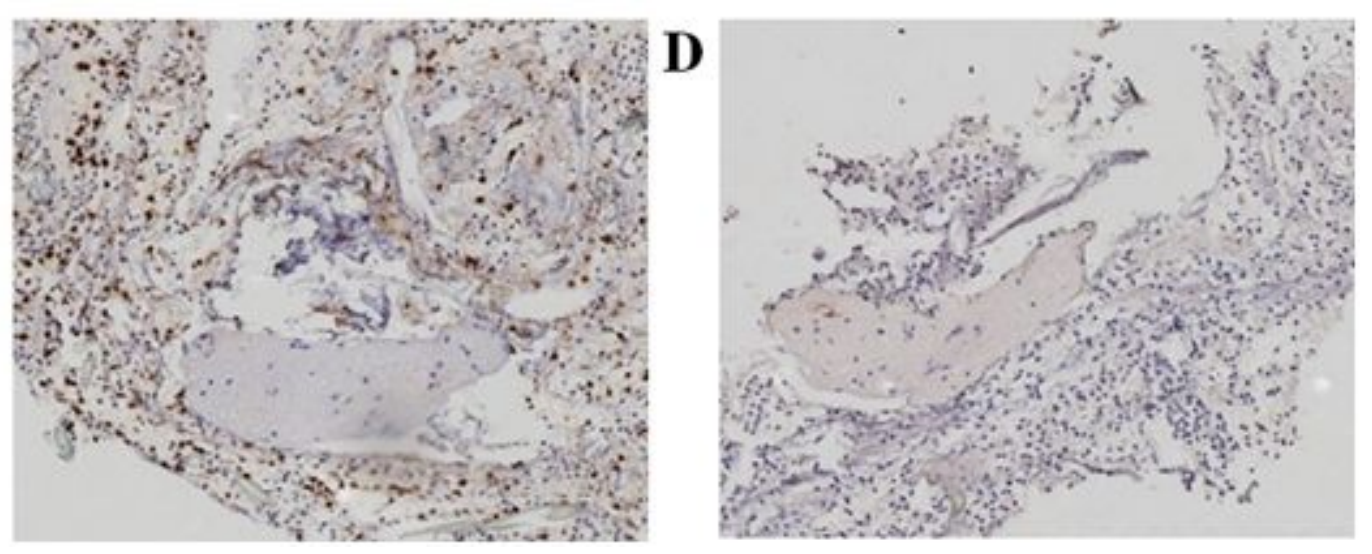

\section{Figure 8}

The postoperative pathological diagnosis was vasculitis. Hematoxylin-eosin staining (HE) shows the histology of isolated vasculitis; immunohistochemistry (IHC) shows the expression of leucocyte common antigen (LCA) and smooth muscle actin (SMA) in isolated vasculitis. (A) HE (40x). (B) HE (100x). (C) IHC of LCA expression (100x). (D) IHC of SMA expression (100x). 

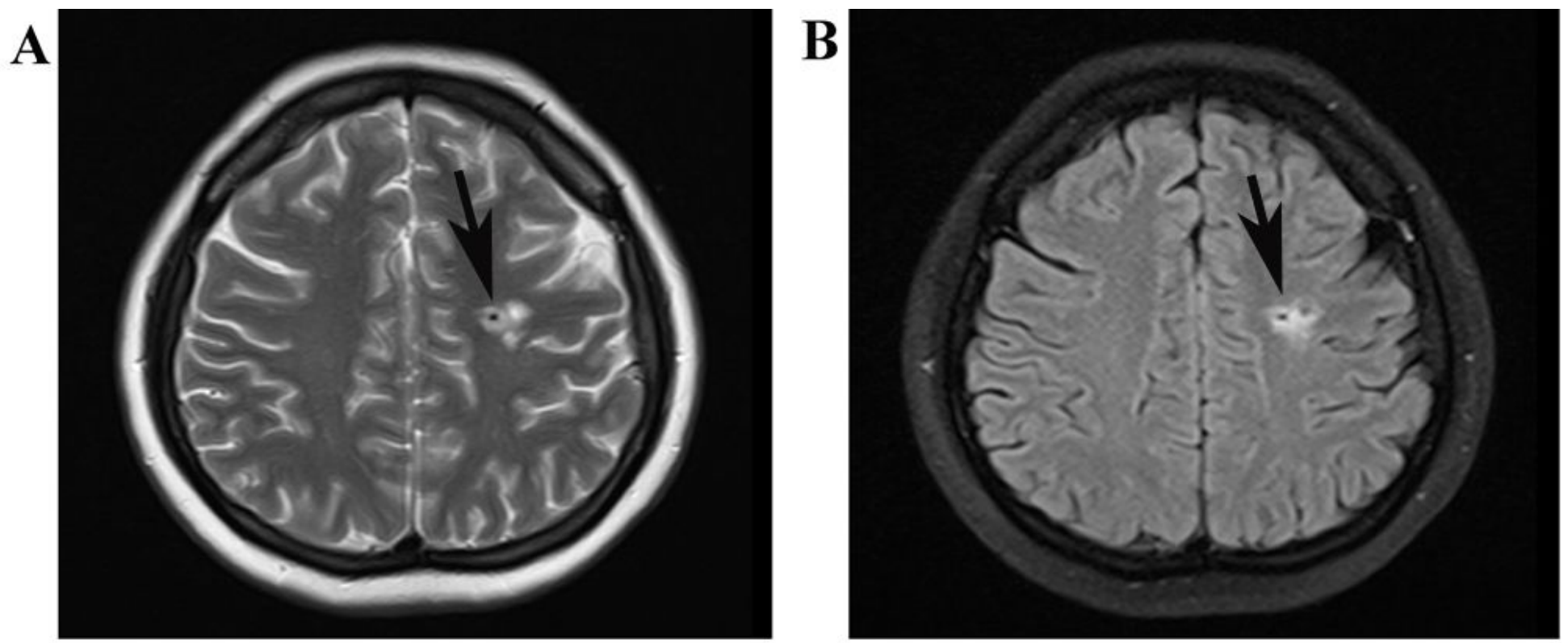

\section{C}

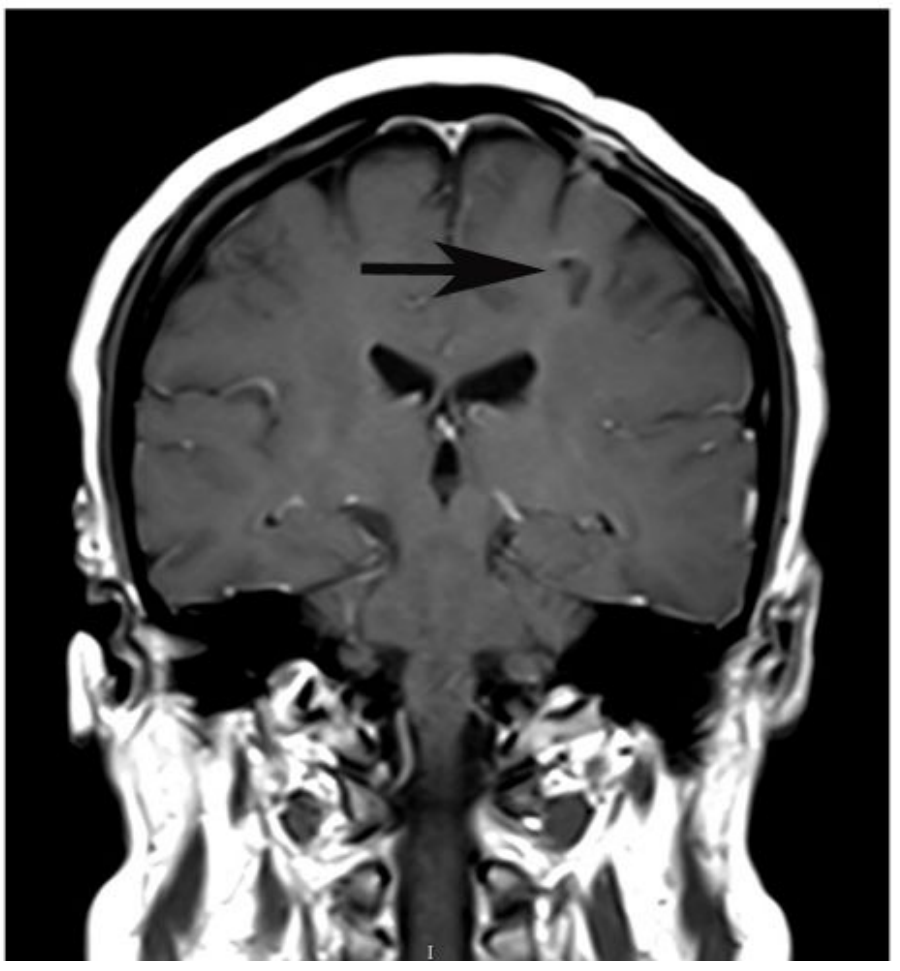

D

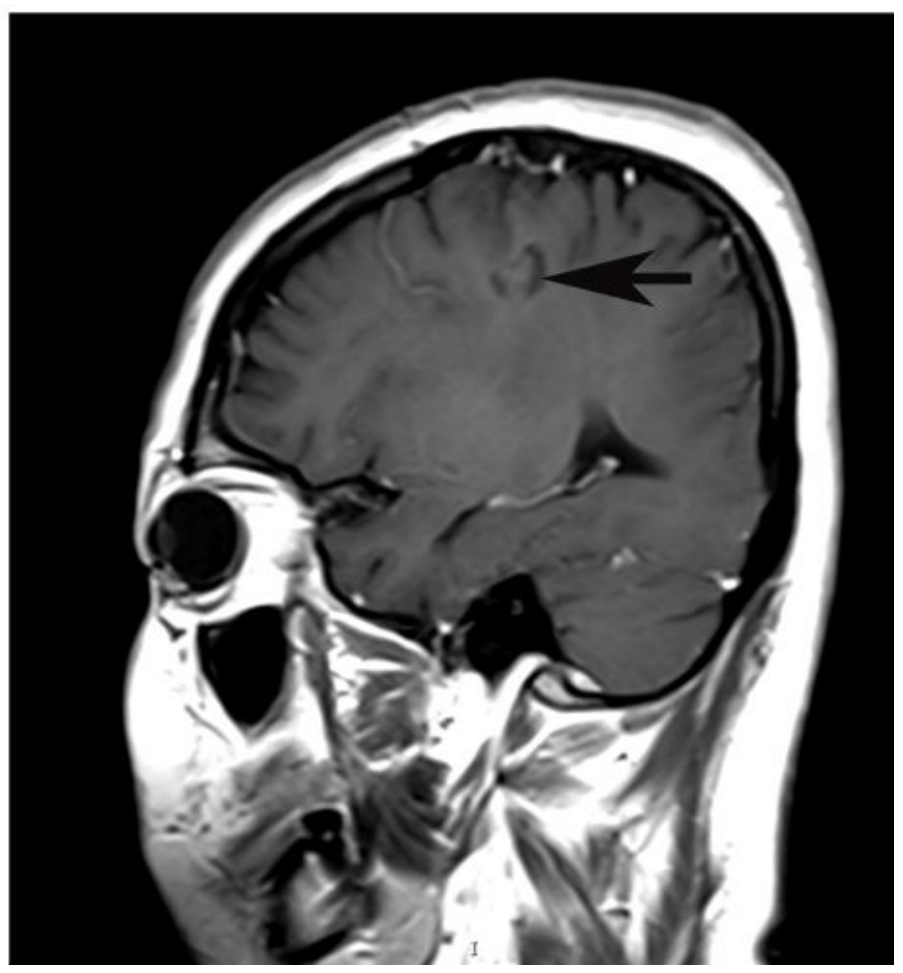

\section{Figure 9}

Magnetic resonance images obtained 2 months after surgery showed a small residual cavity of hypointensity on T1-weighted imaging (T1WI) and hyperintensity on T2-weighted imaging (T2WI) and fluid-attenuated inversion recovery (FLAIR), with a clear boundary in the left centrum semiovale after surgical treatment with no enhancement (arrows point to the center of the focus). (A) T2WI axial view. (B) T2-FLAIR axial view. (C) Contrast enhanced (CE)-T1WI coronal view. (D) CE-T1WI sagittal view.

\section{Supplementary Files}

This is a list of supplementary files associated with this preprint. Click to download. 
- CAREchecklistEnglish2013.pdf

Page 18/18 